\title{
Is type 2 diabetes mellitus a coronary heart disease equivalent or not? Do not just enjoy the debate and forget the patient!
}

\author{
Niki Katsiki ${ }^{1}$, Maciej Banach ${ }^{2,3,4}$, Dimitri P. Mikhailidis ${ }^{5}$
}

\begin{abstract}
${ }^{1}$ First Department of Internal Medicine, Division of Endocrinology and Metabolism, Diabetes Center, Medical School, AHEPA University Hospital, Thessaloniki, Greece ${ }^{2}$ Department of Hypertension, Chair of Nephrology and Hypertension, Medical University of Lodz, Poland

3Polish Mother's Memorial Hospital Research Institute (PMMHRI), Lodz, Poland ${ }^{4}$ Cardiovascular Research Centre, University of Zielona Gora, Zielona Gora, Poland ${ }^{5}$ Department of Clinical Biochemistry, Royal Free Hospital Campus, University College London Medical School, University College London (UCL), London, UK
\end{abstract}

Submitted: 2 September 2019

Accepted: 16 September 2019

Arch Med Sci 2019; 15 (6): 1357-1364

DOI: https://doi.org/10.5114/aoms.2019.89449

Copyright @ 2019 Termedia \& Banach

\begin{abstract}
In the last several years there has been a large debate whether patients with type 2 diabetes (T2DM) should be treated as those with high or very high cardiovascular risk, and whether T2DM should be considered as equivalent to coronary heart disease (CHD). It all started in the 2001 in National Cholesterol Education Program - Adult Treatment Panel III recommendations, and the knowledge has changed on this issue at least several times. But the main problem is that due to these inconsistencies and different approaches to the cardiovascular risk of T2DM patients, we have more and more patients with T2DM not effectively treated, and diabetologists mostly focus on glucose (glucocentric approach), often forgetting about the overall cardiovascular risk of those patients. In this review we discuss the above-mentioned topic, try to give some practical suggestions, and raise the issue of whether we should start a discussion on treating all patients with T2DM as those at very high cardiovascular risk, or to at least to try to unify the definition and find such variables/risk factors which are easy to measure to help physicians to treat those patients optimally. We have obviously discussed these issues in the context of new European Society of Cardiology (ESC)/European Association for the Study of Diabetes (EASD) Guidelines 2019.
\end{abstract}

Key words: CHD equivalent, diabetes, guidelines, prevention, risk stratification.

Type 2 diabetes mellitus (T2DM) is associated with increased prevalence of cardiovascular disease (CVD) [1-5]. Indeed, T2DM patients have a 2-4-fold higher risk for CVD morbidity and mortality than healthy non-diabetics [6]. Furthermore, CVD is the main cause of mortality in T2DM patients, accounting for almost $80 \%$ of deaths $[7,8]$. The association between T2DM and CVD is supported not only by observational data and meta-analyses $[9,10]$, but also has a pathophysiological background based on the CV continuum that characterizes T2DM [11, 12]. The latter involves a chronic state of vascular inflammation, endothelial and platelet dysfunction, induced by hyperglycemia and insulin resistance

\author{
Corresponding authors: \\ Niki Katsiki MSc, PhD, MD, \\ FRSPH \\ First Department \\ of Internal Medicine \\ AHEPA University \\ Hospital \\ 1 Stilponos Kyriakidi St \\ 54621 Thessaloniki, Greece \\ Phone: +30 6980030212 \\ E-mail: nikikatsiki@ \\ hotmail.com \\ Prof. Maciej Banach \\ MD, PhD, FNLA, FAHA, FESC, \\ FASA \\ Department \\ of Hypertension \\ WAM University \\ Hospital in Lodz \\ Medical University of Lodz \\ 113 Zeromskiego St \\ 90-549 Lodz, Poland \\ Phone: +48426393771 \\ Fax: +48 426393771 \\ E-mail: maciejbanach77@ \\ gmail.com
}


that predisposes to macrovascular complications (i.e. CVD) even before T2DM diagnosis [13]. It has been reported that T2DM patients generally have coronary plaques with larger necrotic cores and greater inflammation (with more $\mathrm{T}$ lymphocytes and macrophages) as well as an increased rate of positive remodeling and plaque ruptures compared with nondiabetics controls, thus suggesting a more active atherosclerotic process $[14,15]$.

T2DM and CVD share a common pathogenesis (i.e. oxidative stress, inflammation and atherothrombosis), as well as common risk factors, including obesity, hyperinsulinemia, hypertension, dyslipidemia and non-alcoholic fatty liver disease [16-24]. Indeed, insulin resistance per se represents a major cause of CVD [25]. In this context, even "pre-diabetes" has been linked to increased risk of CHD events, stroke and all-cause death [26, 27]. Of note, there is a link between pre-diabetes, metabolic syndrome (MetS) and CVD risk [28, 29]. Similarly, MetS presence has been associated with both micro- and macrovascular complications in T2DM patients [30-33].

Historically, in 1998, Haffner et al. [34] evaluated the 7-year incidence of fatal or nonfatal myocardial infarction (MI) and stroke in a Finnish population cohort $(n=1,059$ T2DM patients and 1,373 healthy non-diabetics). The 7-year rates of $\mathrm{MI}$ in T2DM patients with and without prior $\mathrm{MI}$ were 45.0 and $20.2 \%$, respectively $(p<0.001$ ); the corresponding rates for nondiabetics were 18.8 and $3.5 \%$, respectively $(p<0.001)$ [34]. Furthermore, the 7-year incidence of stroke in T2DM patients with and without prior MI was 19.5 and $10.3 \%$, respectively $(p<0.001)$; for nondiabetics the corresponding values were 7.2 and $1.9 \%$, respectively ( $p=0.01$ ) [34]. Finally, 7-year CVD death rates were 42.0 and $15.4 \%$ for T2DM patients with and without prior MI $(p<0.001)$, whereas for nondiabetics they were 15.9 and $2.1 \%$, respectively $(p<0.001)$. The 7-year CVD mortality rates remained similar between T2DM patients without prior $\mathrm{MI}$ and nondiabetics with prior $\mathrm{MI}$, even after adjustment for age, gender, smoking, hypertension, triglyceride, high-density lipoprotein cholesterol (HDL-C) and low-density lipoprotein cholesterol (LDL-C) levels [34]. These findings supported the notion that T2DM patients without known CHD have a similar CVD morbidity and mortality risk to nondiabetic CHD patients, thus highlighting the need for aggressive treatment of CVD risk factors in both these patient populations.

There has been a debate on whether T2DM (without known CVD) is a CHD equivalent or not, i.e. whether it is meaningful to consider the term "primary CVD prevention" in the presence of T2DM. In this context, it has been suggested that disease duration is important and that only T2DM patients with long disease duration, i.e. $\geq 10$ years (without known CVD), could be regarded as CHD equivalent patients, thus having a similar risk of future CHD events compared with CHD patients without T2DM [6, 35]. In the 7-year cohort study by Haffner et al. [34] supporting the recognition of T2DM as a CHD equivalent, the mean T2DM duration was 8 years. Furthermore, when this Finnish population cohort was followed up for 18 years, CHD mortality remained similar between T2DM patients without $\mathrm{MI}$ and nondiabetics with prior $\mathrm{MI}$, even after adjustment for several risk factors (i.e. age, gender, smoking, hypertension, total cholesterol, HDL-C, triglycerides) as well as T2DM duration [36]. Nevertheless, there are several studies showing that the earlier the onset and the longer the disease, the higher the CVD risk for T2DM patients $[37,38]$. These findings are reasonable since atherosclerosis is an age-related disease and CVD risk increases with aging. However, the "real" clinical issue is not whether T2DM is or is not a CHD equivalent, but that T2DM patients (even without known CVD) are at a very high CVD risk, especially in the presence of CVD risk factors or target organ damage. This is clinically important since it can influence therapeutic strategies in T2DM patients without "diagnosed" CVD as well as adherence to treatment and clinical inertia [39]. In other words, why wait until a CVD event occurs in these very high-risk patients instead of preventing it?

The real diagnostic and therapeutic problem, however, is associated with the fact that, based on the European Society of Cardiology (ESC) risk categories, T2DM patients without target organ damage or other CVD risk factors are still classified as being at high CVD risk [40]. From the clinical point of view the question is whether we really see such patients in everyday practice. T2DM is usually diagnosed late, and in a large percentage of these patients, subclinical organ damage and/or other concomitant risk factors (e.g. dyslipidemia, overweight/obesity, hypertension) are already present [3]. Thus, while carefully investigating atherosclerosis risk factors and/or parameters of subclinical organ damage (similar to those recommended in patients with hypertension), there are very few T2DM patients who meet the criteria of those only at high CVD risk. So why simply not consider all T2DM patients as at very high CVD risk in order to start optimal, intensive, hypoglycemic, hypolipidemic and hypotensive treatment? We have enough data that such therapy may prevent CVD, being also cost-effective (see text below for details). The therapeutic approach of some diabetologists may still be too glucocentric, thus forgetting that T2DM patients are very likely to suffer a CVD event or death. Also, T2DM patients may be treated by general practitioners (GPs) worldwide, 
with only a small percentage of these patients being under the care of a diabetologist or a cardiologist. Therefore, it is important that all physicians who treat T2DM patients recognize their very high CVD risk.

Regarding recommendations from scientific societies, more than 2 decades ago, in 1993, the Second Report of the National Cholesterol Education Program (NCEP) Expert Panel on Detection, Evaluation, and Treatment of High Blood Cholesterol in Adults (Adult Treatment Panel II) recognized T2DM as a CHD risk factor together with other established CVD risk factors including age, family history of premature CHD, smoking and hypertension [41]. In 2001, the NCEP ATP III stated that "diabetes is regarded as a coronary heart disease risk equivalent" [42]. Currently, as highlighted in a recent special Report from the American Heart Association (AHA)/American College of Cardiology (ACC), history of T2DM is included as a CVD predictor in different risk assessment tools such as the Framingham risk calculator, the ACC risk estimator and the AHA risk calculator [43]. The same Special Report recommends that individuals (without known CVD) with a coronary artery calcium (CAC) score of 0 should be treated with a statin only in the presence of T2DM, heavy cigarette smoking or family history of premature CVD, thus highlighting the clinical significance of T2DM in relation to CVD risk [43]. According to the 2019 ESC/European Association for the Study of Diabetes (EASD) guidelines, CAC scoring may be considered as a CV risk modifier in T2DM patients [44].

The latest (2019) American Diabetes Association (ADA) guidelines consider T2DM (without known CVD) in the presence of other CHD risk factors (including hypertension, smoking, albuminuria, chronic kidney disease (CKD), LDL-C $\geq 100$ $\mathrm{mg} / \mathrm{dl}(2.6 \mathrm{mmol} / \mathrm{l})$ or family history of premature CVD) as a CHD equivalent [45]. Similarly, in the 2016 ESC/European Atherosclerosis Society (EAS) guidelines, T2DM patients (without a history of a CVD event) with target organ damage (e.g. proteinuria) or a major CVD risk factor (e.g. smoking, dyslipidemia or hypertension) are recognized as being at very high risk, i.e. in the same risk category as patients with known CVD [40]. Of note, left ventricular hypertrophy (LVH) represents another target organ damage that has been linked to CVD and renal dysfunction in T2DM patients [46-48]. However, the latest (2019) ESC/EAS guidelines categorize T2DM patients (without known CVD) with target organ damage (defined as microalbuminuria, retinopathy or neuropathy) or at least three major risk factors as at very high risk [49]. Similarly, in the 2019 ESC/EASD guidelines T2DM patients (without CVD history) with target organ damage (defined as proteinuria, eGFR $\geq 30 \mathrm{ml} / \mathrm{min} /$
$1.73 \mathrm{~m}^{2}, \mathrm{LVH}$ or retinopathy) or at least three major risk factors (i.e. hypertension, dyslipidemia, smoking, obesity, age) are categorized as at very high risk [44]. Table I summarizes the recommendations from scientific societies regarding T2DM and CVD risk.

The ADA guidelines do not recommend screening for CHD in asymptomatic T2DM patients, partly because these patients are already at high risk and thus should be treated with intensive medical therapy [45]. These recommendations consider further investigations for $\mathrm{CHD}$ in the presence of electrocardiogram (ECG) abnormalities (e.g. Q waves), atypical cardiac symptoms (e.g. chest discomfort or unexplained dyspnea), carotid bruits, stroke, transient ischemic attack, claudication or peripheral artery disease (PAD). The critical question is how many patients with diagnosed T2DM indeed undergo this diagnostic process worldwide. A previous (2013) joint Task Force of the ESC/ADA recommends the performance of ECG, echocardiography, exercise test and Holter monitoring in T2DM patients without known CVD, based on clinical judgment [13]. In the 2019 ESC/ EASD guidelines, a resting ECG is recommended in T2DM patients with hypertension or suspected CVD [45]. Of note, up to $60 \%$ of MIs in T2DM patients may be asymptomatic, thus being diagnosed only by ECG screening [13]. The prevalence of silent myocardial ischemia (SMI) is $20-35 \%$ in T2DM patients with additional CVD risk factors, and up to $70 \%$ of patients with SMI may also have significant coronary stenoses [13]. Furthermore, T2DM is related to an increased risk for sudden cardiac death [50, 51] and atrial fibrillation [52, 53]. With regard to peripheral arteries, the 2019 ESC/ EASD guidelines consider the assessment of carotid (and/or femoral) plaque burden by ultrasonography as a risk modifier in asymptomatic T2DM patients [44]. In contrast, surprisingly, carotid intima-media thickness is not recommended by these guidelines for $\mathrm{CV}$ risk evaluation in T2DM patients [44].

Therefore, there is an urgent need to better define CHD screening in T2DM patients. A comprehensive foot evaluation should be performed at least annually in all T2DM patients, including measurement of the ankle-brachial index (ABI), especially in patients with decreased or absent pedal pulses or symptoms of claudication [45]. Of note, apart from a diagnostic tool for $P A D$, the $A B I$ is considered a useful marker adding predictive value to the usual CVD risk estimation as supported by the ESC Working Group on peripheral circulation [54]. Since PAD is mostly asymptomatic (and thus frequently remains undiagnosed and untreated) and T2DM patients are more prone to PAD than the general population [55], $A B I$ measurement should 
Table I. Summary of recommendations from scientific societies regarding type 2 diabetes mellitus and cardiovascular risk

\begin{tabular}{|c|c|}
\hline Scientific societies (year) [reference] & Recommendations \\
\hline NCEP ATP III (2001) [42] & DM is a CHD risk equivalent \\
\hline \multirow[t]{2}{*}{ AHA/ACC Special Report (2019) [43] } & $\begin{array}{l}\text { Type } 2 \text { DM is included as a CVD predictor in different risk assessment tools } \\
\text { such as the Framingham risk calculator, the ACC risk estimator and the AHA } \\
\text { risk calculator }\end{array}$ \\
\hline & $\begin{array}{l}\text { In the presence of type } 2 \mathrm{DM} \text {, heavy cigarette smoking or family history } \\
\text { of premature CVD, patients with CAC score } 0 \text { should be treated with a statin }\end{array}$ \\
\hline ADA (2019) [44] & $\begin{array}{l}\text { DM patients with multiple coronary risk factors have a risk equivalent to } \\
\text { that of patients with ASCVD }\end{array}$ \\
\hline ESC/EAS (2016) [40] & $\begin{array}{l}\text { Type } 2 \text { DM patients (without a history of CVD event) with target organ dam- } \\
\text { age (e.g. proteinuria) or a major CVD risk factor (e.g. smoking, dyslipidemia } \\
\text { or hypertension) are at very high risk, i.e. in the same risk category as pa- } \\
\text { tients with known CVD }\end{array}$ \\
\hline ESC/EAS (2019) [49] & $\begin{array}{l}\text { Type } 2 \text { DM patients (without a history of a CVD event) with target organ } \\
\text { damage (microalbuminuria, retinopathy, neuropathy) or at least three major } \\
\text { risk factors are at very high risk, i.e. in the same risk category as patients } \\
\text { with known CVD }\end{array}$ \\
\hline ESC/EASD (2019) [44] & $\begin{array}{l}\text { Type } 2 \text { DM patients (without a history of a CVD event) with target organ } \\
\text { damage (proteinuria, eGFR } \geq 30 \mathrm{ml} / \mathrm{min} / 1.73 \mathrm{~m}^{2} \text {, left ventricular hypertro- } \\
\text { phy, retinopathy) or at least three major risk factors (hypertension, dyslip- } \\
\text { idemia, smoking, obesity, age) are at very high risk, i.e. in the same risk } \\
\text { category as patients with known CVD }\end{array}$ \\
\hline \multicolumn{2}{|c|}{$\begin{array}{l}\text { ICEP ATP III - Third Report of the National Cholesterol Education Program (NCEP) Expert Panel on Detection, Evaluation, and Treatment } \\
\text { fHigh Blood Cholesterol in Adults (Adult Treatment Panel III), AHA - American Heart Association, ACC - American College of Cardiology, } \\
D A-A m e r i c a n \text { Diabetes Association, EAS - European Atherosclerosis Society, ESC - European Society of Cardiology, DM - diabetes mellitus, } \\
\text { VD - cardiovascular disease, CHD - coronary heart disease, CAC - coronary artery calcium, ASCVD - atherosclerotic cardiovascular } \\
\text { isease, eGFR - estimated glomerular filtration rate. }\end{array}$} \\
\hline
\end{tabular}

be performed in T2DM patients, as recommended by the joint 2013 Task Force of the ESC/ADA [13] and the 2019 ESC/EASD guidelines [44]. It should be noted that T2DM patients might frequently have high $A B I$ values (i.e. > 1.3), mainly due to medial artery calcification, a characteristic feature of T2DM [56]. Such elevated $A B I$ values also correlate with CVD morbidity and death [56], as also highlighted by the 2019 ESC/EASD guidelines [44]. According to current AHA/ACC [57] and ESC/EASD guidelines [44], the toe-brachial index (or Duplex ultrasound [44]) should be measured in such patients with non-compressible arteries to diagnose PAD.

Multifactorial treatment is critical in T2DM patients (with or without known CVD). The Steno-2 study in 2008 showed that multifactorial intervention (targeting glucose, blood pressure, lipids and platelets) significantly reduced CVD events and death as well as total mortality in 160 T2DM patients with microalbuminuria [58]. Another recent cohort study ( $n=144,271$ T2DM patients without history of (VD) found that the greatest reduction in CVD events was achieved when hemoglobin $A_{1 c}$, blood pressure and LDL-C were all optimally controlled [59]. The 2019 ESC/EASD guidelines mention that the combined reduction of $\mathrm{HbA}_{1 \mathrm{c}}$, systolic blood pressure and lipids can decrease CVD events by $75 \%$, and that multifactorial treatment remains underused [44].
Regarding T2DM patients without known CVD, statins can prevent CVD events [60], as shown in the Collaborative Atorvastatin Diabetes Study (CARDS) [61], the Heart Protection Study (HPS) [62] and the Anglo-Scandinavian Cardiac Outcomes Trial (ASCOT) Lipid Lowering Arm (LLA) [63]. This is also supported by available meta-analyses $[64,65]$. In terms of antihypertensive drugs, a fixed combination of perindopril and indapamide was reported to significantly lower the rate of microand macrovascular events in T2DM patients, irrespectively of the presence or absence of CVD at baseline in the Action in Diabetes and Vascular disease: preterAx and diamicroN-MR Controlled Evaluation (ADVANCE) trial [66]. Ramipril also significantly decreased CVD morbidity and mortality as well as all-cause death in T2DM patients with or without known CVD in the Heart Outcomes Prevention Evaluation (HOPE) study [67]. Similar results were obtained with losartan in T2DM patients with LVH (but without known CVD) in the Losartan Intervention For Endpoint reduction (LIFE) study [68]. Of note, fixed combinations of antihypertensive drugs and/or polypills containing aspirin, statin and one or more antihypertensive medication may represent a cost-effective strategy for CVD prevention, also improving patients' adherence to treatment and thus achievement of therapeutic goals [69-71]. 
With regard to antidiabetic drugs, in the recently published Researching Cardiovascular Events with a Weekly Incretin in Diabetes (REWIND) trial [72], dulaglutide, a glucagon-like peptide-1 receptor agonist (GLP-1 RA) administered subcutaneously once weekly, was shown to significantly reduce the composite endpoint of non-fatal MI, non-fatal stroke or CVD death compared with placebo in both groups of T2DM patients (i.e. with known CVD (31.5\%) or with CVD risk factors); this benefit was mainly attributed to a decrease in non-fatal stroke occurrence. Semaglutide, another subcutaneously administered GLP-1 RA once weekly, significantly lowered the rates of the primary outcome (MI, stroke or CVD mortality) compared with placebo in T2DM patients with or without known CVD in the Trial to Evaluate Cardiovascular and Other Long-term Outcomes with Semaglutide in Subjects with Type 2 Diabetes (SUSTAIN-6) [73]; the observed benefit was largely attributed to stroke prevention. In subgroup analyses, the semaglutide-related reduction of the composite endpoint was similar in T2DM patients with known CVD or with CVD risk factors only (17\% of the total study population [73].

Among sodium-glucose co-transporter-2 inhibitors (SGLT2i), canagliflozin was reported to significantly decrease the composite of CVD morbidity and mortality, as well as hospitalization for heart failure (HF), compared with placebo in T2DM patients in the Canagliflozin Cardiovascular Assessment Study (CANVAS) program [74]. Of note, subgroup analyses showed a similar CVD benefit between T2DM patients with (65.6\%) or without a history of CVD at baseline [74]. However, canagliflozin use was related to a doubled risk of amputation in the lower extremities compared with placebo. This side effect was observed only with canagliflozin and not with other SGLT2i (e.g. empagliflozin or dapagliflozin), and thus it should be taken into consideration by physicians who treat T2DM patients $[45,75]$. In the Dapagliflozin Effect on Cardiovascular Events-Thrombolysis in Myo- cardial Infarction 58 (DECLARE-TIMI 58) trial [76], dapagliflozin significantly lowered the rate of $\mathrm{HF}$ hospitalization compared with placebo in T2DM patients with (40.6\%) or without known CVD, as shown in subgroup analyses.

The BI 10773 (Empagliflozin) Cardiovascular Outcome Event Trial in Type 2 Diabetes Mellitus Patients (EMPA-REG OUTCOME) [77] was the first positive CVD outcome trial with a SGLT2i, thus introducing a new era in the treatment of T2DM patients. In this trial, empagliflozin use was associated with a significant reduction in the composite of CVD morbidity and mortality, as well as HF hospitalizations, but also significantly decreased CVD and all-cause death compared with placebo in T2DM patients with known CVD at baseline [77]. Interestingly, all empagliflozin-related CVD benefits (i.e. composite of CVD morbidity and death, as well as HF hospitalization, total and CVD mortality) were consistent in patients with or without a prior atherothrombotic event at baseline (i.e. stroke or MI) [78], thus highlighting its clinical usefulness in T2DM patients without known MI or stroke. Of note, a report from the ESC Cardiovascular Roundtable in 2018 [79], as well as the 2019 ESC/EASD guidelines, recommends the use of SGLT2i or GLP-1 RAs with proven CVD benefit as monotherapy in drug-naïve T2DM patients with known CVD or at high/very high risk [44].

Based on the above, T2DM patients should be individually evaluated, in terms of CVD risk stratification, regarding CVD family history (especially with early onset), hypertension, dyslipidemia, smoking, obesity, CKD, albuminuria, LVH, claudication, abnormal ABI, carotid bruits (and carotid ultrasound) and abnormal ECG changes (Figure 1). It is however a great challenge to select those risk factors which should be relatively easy to measure and evaluate also for the non-specialist, such as GPs or internal medicine physicians, especially in the light of knowledge of limited diabetologists' accessibility for T2DM patients. In the presence of such CVD risk factors, T2DM should be treated as

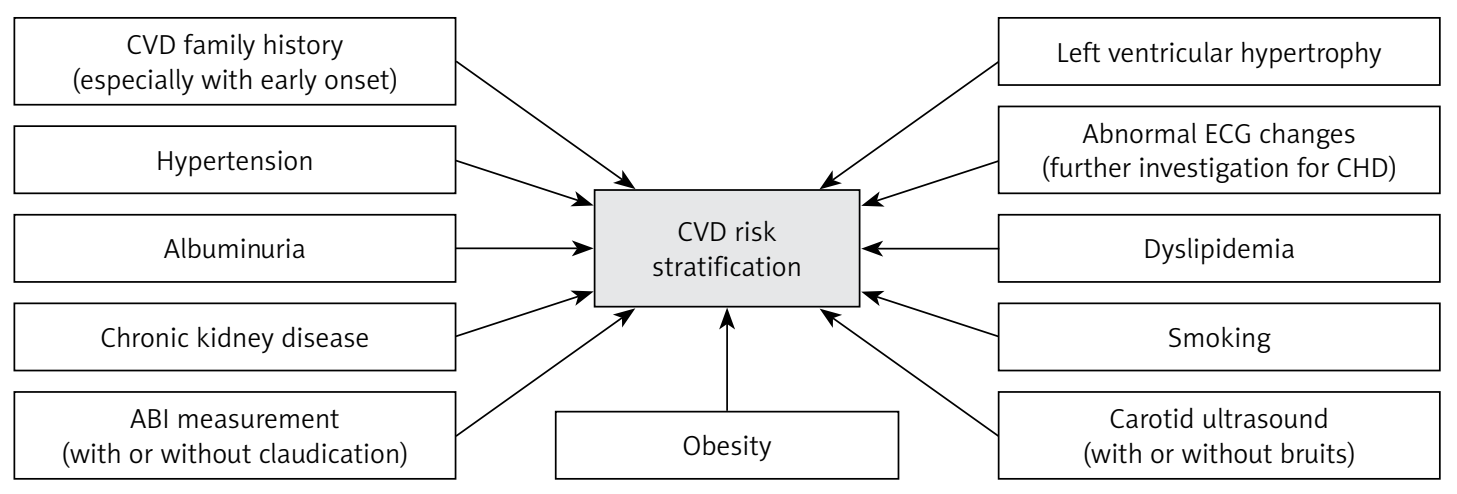

Figure 1. Evaluation of patients with type 2 diabetes mellitus in terms of cardiovascular risk stratification $C V D$ - cardiovascular disease, CHD - coronary heart disease, ABI - ankle-brachial index, ECG - electrocardiogram. 
a CHD equivalent, irrespective of disease duration. It follows that in such patients all CVD risk factors should be aggressively treated. Furthermore, the selection of antidiabetic drugs should focus on preventing CVD morbidity and mortality.

\section{Conflict of interest}

NK has given talks, attended conferences and participated in trials sponsored by Amgen, Astra Zeneca, Boehringer Ingelheim, Elpen, Mylan, NovoNordisk, PharmaSwiss, Sanofi, Servier and WinMedica. DPM has given talks and attended conferences sponsored by Amgen, AstraZeneca and Libytec. MB has received research grants/ support from Sanofi and Valeant, and has served as a consultant for Abbott/Mylan, Akcea, Amgen, KRKA, NovoNordisk, MSD, Polfarmex, Polpharma, Sanofi-Aventis, Servier, Esperion, and Resverlogix.

\section{References}

1. Katsiki N, Mikhailidis DP, Banach M. Leptin, cardiovascular diseases and type 2 diabetes mellitus. Acta Pharmacol Sin 2018; 39: 1176-88.

2. Einarson TR, Acs A, Ludwig C, Panton UH. Prevalence of cardiovascular disease in type 2 diabetes: a systematic literature review of scientific evidence from across the world in 2007-2017. Cardiovasc Diabetol 2018; 17: 83.

3. Katsiki N, Purrello F, Tsioufis C, Mikhailidis DP. Cardiovascular disease prevention strategies for type 2 diabetes mellitus. Expert Opin Pharmacother 2017; 18: 1243-60.

4. Norhammar A, Bodegård J, Nyström T, Thuresson $M$, Eriksson JW, Nathanson D. Incidence, prevalence and mortality of type 2 diabetes requiring glucose-lowering treatment, and associated risks of cardiovascular complications: a nationwide study in Sweden, 2006-2013. Diabetologia 2016; 59: 1692-701.

5. Katsiki N, Mikhailidis DP. Management of patients with type 2 diabetes mellitus and acute coronary syndrome: better be safe than sorry! J Diabetes Complications 2019; 33: 465-7.

6. Bertoluci MC, Rocha VZ. Cardiovascular risk assessment in patients with diabetes. Diabetol Metab Syndr 2017 9: 25

7. Abdul-Ghani M, DeFronzo RA, Del Prato S, Chilton R, Singh R, Ryder REJ. Cardiovascular disease and type 2 diabetes: has the dawn of a new era arrived? Diabetes Care 2017; 40: 813-20.

8. Papatheodorou K, Banach M, Bekiari E, Rizzo M, Edmonds M. Complications of diabetes 2017. J Diabetes Res 2018; 2018: 3086167.

9. Emerging Risk Factors Collaboration, Sarwar N, Gao P, Seshasai SR, et al.; Diabetes mellitus, fasting blood glucose concentration, and risk of vascular disease: a collaborative meta-analysis of 102 prospective studies. Lancet 2010; 375: 2215-22.

10. Huxley R, Barzi F, Woodward M. Excess risk of fatal coronary heart disease associated with diabetes in men and women: meta-analysis of 37 prospective cohort studies. BMJ 2006; 332: 73-8.

11. Paneni F, Costantino S, Cosentino F. Insulin resistance, diabetes, and cardiovascular risk. Curr Atheroscler Rep 2014; 16: 419 .
12. Orasanu G, Plutzky J. The pathologic continuum of diabetic vascular disease. J Am Coll Cardiol 2009; 53 (5 Suppl): S35-42.

13. Rydén L, Grant PJ, Anker SD, et al.; ESC Committee for Practice Guidelines (CPG). ESC Guidelines on diabetes, pre-diabetes, and cardiovascular diseases developed in collaboration with the EASD: the Task Force on diabetes, pre-diabetes, and cardiovascular diseases of the European Society of Cardiology (ESC) and developed in collaboration with the European Association for the Study of Diabetes (EASD). Eur Heart J 2013; 34: 3035-87.

14. Yahagi K, Kolodgie FD, Lutter C, et al. Pathology of human coronary and carotid artery atherosclerosis and vascular calcification in diabetes mellitus. Arterioscler Thromb Vasc Biol 2017; 37: 191-204.

15. Mazidi M, Toth PP, Banach M. C-reactive protein is associated with prevalence of the metabolic syndrome, hypertension, and diabetes mellitus in US adults. Angiology 2018; 69: 438-42.

16. Meigs JB. Epidemiology of type 2 diabetes and cardiovascular disease: translation from population to prevention: the Kelly West award lecture 2009. Diabetes Care 2010; 33: 1865-71.

17. Matheus AS, Tannus LR, Cobas RA, Palma CC, Negrato CA, Gomes MB. Impact of diabetes on cardiovascular disease: an update. Int J Hypertens 2013; 2013: 653789.

18. Katsiki N, Tentolouris N, Mikhailidis DP. Dyslipidaemia in type 2 diabetes mellitus: bad for the heart. Curr Opin Cardiol 2017; 32: 422-9.

19. Katsiki N, Perez-Martinez P, Anagnostis P, Mikhailidis DP, Karagiannis A. Is nonalcoholic fatty liver disease indeed the hepatic manifestation of metabolic syndrome? Curr Vasc Pharmacol 2018; 16: 219-27.

20. Athyros VG, Tziomalos K, Katsiki N, Doumas M, Karagiannis A, Mikhailidis DP. Cardiovascular risk across the histological spectrum and the clinical manifestations of non-alcoholic fatty liver disease: an update. World J Gastroenterol 2015; 21: 6820-34.

21. Katsiki N, Athyros VG, Karagiannis A, Wierzbicki AS, Mikhailidis DP. Should we expand the concept of coronary heart disease equivalents? Curr Opin Cardiol 2014; 29: 389-95.

22. Mullish BH, Forlano R, Manousou P, Mikhailidis DP. Non-alcoholic fatty liver disease and cardiovascular risk: an update. Expert Rev Gastroenterol Hepatol 2018; 12: 1175-7.

23. Katsiki N, Athyros VG, Mikhailidis DP. Non-alcoholic fatty liver disease in patients with type 2 diabetes mellitus: effects of statins and antidiabetic drugs. J Diabetes Complications 2017; 31: 521-2.

24. Athyros VG, Alexandrides TK, Bilianou $\mathrm{H}$, et al. The use of statins alone, or in combination with pioglitazone and other drugs, for the treatment of non-alcoholic fatty liver disease/non-alcoholic steatohepatitis and related cardiovascular risk. An Expert Panel Statement. Metabolism 2017; 71: 17-32.

25. Adeva-Andany MM, Martínez-Rodríguez J, GonzálezLucán M, Fernández-Fernández C, Castro-Quintela E. Insulin resistance is a cardiovascular risk factor in humans. Diabetes Metab Syndr 2019; 13: 1449-55.

26. Brannick B, Dagogo-Jack S. Prediabetes and cardiovascular disease: pathophysiology and interventions for prevention and risk reduction. Endocrinol Metab Clin North Am 2018; 47: 33-50.

27. Huang Y, Cai X, Mai W, Li M, Hu Y. Association between prediabetes and risk of cardiovascular disease and all cause mortality: systematic review and meta-analysis. BMJ 2016; 355: i5953. 
28. Mayans L. Metabolic syndrome: insulin resistance and prediabetes. FP Essent 2015; 435: 11-6.

29. Grundy SM. Pre-diabetes, metabolic syndrome, and cardiovascular risk. J Am Coll Cardiol 2012; 59: 635-43.

30. Katsiki N, Anagnostis P, Kotsa K, Goulis DG, Mikhailidis DP. Obesity, metabolic syndrome and the risk of microvascular complications in patients with diabetes mellitus. Curr Pharm Des 2019; 25: 2051-9.

31. Katsiki N, Athyros VG, Karagiannis A, Mikhailidis DP. Metabolic syndrome and non-cardiac vascular diseases: an update from human studies. Curr Pharm Des 2014; 20: 4944-52.

32. Yao MF, He J, Sun X, et al. Gender differences in risks of coronary heart disease and stroke in patients with type 2 diabetes mellitus and their association with metabolic syndrome in China. Int J Endocrinol 2016; 2016: 8483405.

33. Kwasny C, Manuwald U, Kugler J, Rothe U. Systematic review of the epidemiology and natural history of the metabolic vascular syndrome and its coincidence with type 2 diabetes mellitus and cardiovascular diseases in different European countries. Horm Metab Res 2018; 50: 201-8.

34. Haffner SM, Lehto S, Rönnemaa T, Pyörälä K, Laakso M. Mortality from coronary heart disease in subjects with type 2 diabetes and in nondiabetic subjects with and without prior myocardial infarction. N Engl J Med 1998; 339: 229-34.

35. Rana JS, Liu JY, Moffet HH, Jaffe M, Karter AJ. Diabetes and prior coronary heart disease are not necessarily risk equivalent for future coronary heart disease events. J Gen Intern Med 2016; 31: 387-93.

36. Juutilainen A, Lehto S, Rönnemaa T, Pyörälä K, Laakso M. Type 2 diabetes as a "coronary heart disease equivalent": an 18-year prospective population-based study in Finnish subjects. Diabetes Care 2005; 28: 2901-7.

37. Huo X, Gao L, Guo L, et al. Risk of non-fatal cardiovascular diseases in early-onset versus late-onset type 2 diabetes in China: a cross-sectional study. Lancet Diabetes Endocrinol 2016; 4: 115-24.

38. Kianoush S, Al Rifai M, Whelton SP, et al. Stratifying cardiovascular risk in diabetes: the role of diabetes-related clinical characteristics and imaging. I Diabetes Complications 2016; 30: 1408-15.

39. Giugliano D, Maiorino MI, Bellastella G, Esposito K. Diabetes is a cardiovascular disease, isn't it? Diabetes Res Clin Pract 2018; 135: 229-31.

40. Authors/Task Force Members; Catapano AL, Graham I, De Backer G, et al. 2016 ESC/EAS Guidelines for the Management of Dyslipidaemias: The Task Force for the Management of Dyslipidaemias of the European Society of Cardiology (ESC) and European Atherosclerosis Society (EAS) Developed with the special contribution of the European Assocciation for Cardiovascular Prevention \& Rehabilitation (EACPR). Atherosclerosis 2016; 253: 281-344

41. Summary of the second report of the National Cholesterol Education Program (NCEP) Expert Panel on detection, evaluation, and treatment of high blood cholesterol in adults (Adult Treatment Panel II). JAMA 1993; 269: 3015-23.

42. Expert Panel on Detection, Evaluation, and Treatment of High Blood Cholesterol in Adults. Executive Summary of The Third Report of The National Cholesterol Education Program (NCEP) Expert Panel on Detection, Evaluation, And Treatment of High Blood Cholesterol In Adults (Adult Treatment Panel III). JAMA 2001; 285: 2486-97.
43. Lloyd-Jones DM, Braun LT, Ndumele CE, et al. Use of risk assessment tools to guide decision-making in the primary prevention of atherosclerotic cardiovascular disease: a special report from the American Heart Association and American College of Cardiology. J Am Coll Cardiol 2019; 73: 3153-67.

44. Cosentino F, Grant PJ, Aboyans V, et al.; ESC Scientific Document Group, 2019 ESC Guidelines on diabetes, pre-diabetes, and cardiovascular diseases developed in collaboration with the EASD: The Task Force for diabetes, pre-diabetes, and cardiovascular diseases of the European Society of Cardiology (ESC) and the European Association for the Study of Diabetes (EASD). Eur Heart J 2019; doi: 10.1093/eurheartj/ehz486

45. Cardiovascular disease and risk management: standards of medical care in diabetes. 2019 Diabetes Care 2019; 42 (Suppl. 1): S103-23.

46. de Simone G, Wang W, Best LG, et al. Target organ damage and incident type 2 diabetes mellitus: the Strong Heart Study. Cardiovasc Diabetol 2017; 16: 64.

47. Cea-Calvo L, Conthe P, Gómez-Fernández P, de Alvaro F, Fernández-Pérez C; RICARHD investigators. Target organ damage and cardiovascular complications in patients with hypertension and type 2 diabetes in Spain: a cross-sectional study. Cardiovasc Diabetol 2006; 5: 23.

48. Sosner P, Hulin-Delmotte C, Saulnier PJ, et al.; SURDIAGENE Study Group. Cardiovascular prognosis in patients with type 2 diabetes: contribution of heart and kidney subclinical damage. Am Heart J 2015; 169: 108-14.

49. Mach F, Baigent C, Catapano AL, et al.; ESC Scientific Document Group. 2019 ESC/EAS Guidelines for the management of dyslipidaemias: lipid modification to reduce cardiovascular risk: The Task Force for the management of dyslipidaemias of the European Society of Cardiology (ESC) and European Atherosclerosis Society (EAS). Eur Heart J 2019 doi: 10.1093/eurheartj/ehz455

50. Vasiliadis I, Kolovou G, Mavrogeni S, Nair DR, Mikhailidis DP. Sudden cardiac death and diabetes mellitus. J Diabetes Complications 2014; 28: 573-9.

51. Siscovick DS, Sotoodehnia N, Rea TD, Raghunathan TE, Jouven X, Lemaitre RN. Type 2 diabetes mellitus and the risk of sudden cardiac arrest in the community. Rev Endocr Metab Disord 2010; 11: 53-9.

52. Plitt A, McGuire DK, Giugliano RP. atrial fibrillation, type 2 diabetes, and non-vitamin $\mathrm{K}$ antagonist oral anticoagulants: a review. JAMA Cardiol 2017; 2: 442-8.

53. Tadic M, Cuspidi C. Type 2 diabetes mellitus and atrial fibrillation: from mechanisms to clinical practice. Arch Cardiovasc Dis 2015; 108: 269-76.

54. Vlachopoulos C, Xaplanteris P, Aboyans V, et al. The role of vascular biomarkers for primary and secondary prevention. A position paper from the European Society of Cardiology Working Group on peripheral circulation: Endorsed by the Association for Research into Arterial Structure and Physiology (ARTERY) Society. Atherosclerosis 2015; 241: 507-32.

55. Criqui MH, Aboyans V. Epidemiology of peripheral artery disease. Circ Res 2015; 116:1509-26.

56. Rac-Albu M, Iliuta L, Guberna SM, Sinescu C. The role of ankle-brachial index for predicting peripheral arterial disease. Maedica (Buchar) 2014; 9: 295-302.

57. Gerhard-Herman MD, Gornik HL, Barrett C, et al. 2016 AHA/ACC Guideline on the Management of Patients With Lower Extremity Peripheral Artery Disease: A Report of the American College of Cardiology/American Heart Association Task Force on Clinical Practice Guidelines. J Am Coll Cardiol 2017; 69: e71-126. 
58. Gaede P, Lund-Andersen H, Parving HH, Pedersen O. Effect of a multifactorial intervention on mortality in type 2 diabetes. N Engl J Med 2008; 358: 580-91.

59. Wan EYF, Fung CSC, Yu EYT, et al. Effect of multifactorial treatment targets and relative importance of hemoglobin A1c, blood pressure, and low-density lipoprotein-cholesterol on cardiovascular diseases in Chinese primary care patients with type 2 diabetes mellitus: a population-based retrospective cohort study. J Am Heart Assoc 2017; 6: e006400.

60. Katsiki N, Athyros VG, Karagiannis A, Mikhailidis DP. The role of statins in the treatment of type 2 diabetes mellitus: an update. Curr Pharm Des 2014; 20: 3665-74.

61. Colhoun HM, Betteridge DJ, Durrington PN, et al.; CARDS Investigators. Primary prevention of cardiovascular disease with atorvastatin in type 2 diabetes in the Collaborative Atorvastatin Diabetes Study (CARDS): multicentre randomised placebo-controlled trial. Lancet 2004; 364 . 685-96.

62. Heart Protection Study Collaborative Group. MRC/BHF Heart Protection Study of cholesterol-lowering with simvastatin in 5963 people with diabetes: a randomised placebo-controlled trial. Lancet 2003; 361: 2005-16.

63. Sever PS, Poulter NR, Dahlöf B, et al. Reduction in cardiovascular events with atorvastatin in 2,532 patients with type 2 diabetes: Anglo-Scandinavian Cardiac Outcomes Trial--lipid-lowering arm (ASCOT-LLA). Diabetes Care 2005; 28: 1151-7.

64. Cholesterol Treatment Trialists' (CTT) Collaborators. Efficacy and safety of cholesterol-lowering treatment: prospective meta-analysis of data from 90,056 participants in 14 randomised trials of statins. Lancet 2005 ; 366: 1267-78.

65. Costa J, Borges M, David C, Vaz Carneiro A. Efficacy of lipid lowering drug treatment for diabetic and non-diabetic patients: meta-analysis of randomized controlled trials. BMJ 2006; 332: 1115-24.

66. Patel A; ADVANCE Collaborative Group, MacMahon S, Chalmers J, Neal B, et al. Effects of a fixed combination of perindopril and indapamide on macrovascular and microvascular outcomes in patients with type 2 diabetes mellitus (the ADVANCE trial): a randomised controlled trial. Lancet 2007; 370: 829-40.

67. Heart Outcomes Prevention Evaluation Study Investigators. Effects of ramipril on cardiovascular and microvascular outcomes in people with diabetes mellitus: results of the HOPE study and MICRO-HOPE substudy. Lancet 2000; 355: 253-9.

68. Egan B, Gleim G, Panish J. Use of losartan in diabetic patients in the primary care setting: review of the results in LIFE and RENAAL. Curr Med Res Opin 2004; 20: 1909-17.

69. Franczyk B, Gluba-Brzózka A, Jurkiewicz $Ł$, Penson P, Banach M, Rysz J. Embracing the polypill as a cardiovascular therapeutic: is this the best strategy? Expert Opin Pharmacother 2018; 19: 1857-65.

70. Kolte D, Aronow WS, Banach M. Polypills for the prevention of cardiovascular diseases. Expert Opin Investig Drugs 2016; 25: 1255-64.

71. Lopatowska P, Mlodawska E, Tomaszuk-Kazberuk A, Banach M, Malyszko J. Adhering to the principles of clinical pharmacology - the correct fixed combinations of antihypertensive drugs. Expert Rev Clin Pharmacol 2018; 11: 165-70.

72. Gerstein HC, Colhoun HM, Dagenais GR, et al.; REWIND Investigators. Dulaglutide and cardiovascular outcomes in type 2 diabetes (REWIND): a double-blind, random ized placebo-controlled trial. Lancet 2019; 394: 121-30.

73. Marso SP, Bain SC, Consoli A, et al.; SUSTAIN-6 Inves tigators. Semaglutide and cardiovascular outcomes in patients with type 2 diabetes. N Engl J Med 2016; 375: 1834-44.

74. Neal B, Perkovic V, Mahaffey KW, et al.; CANVAS Program Collaborative Group. Canagliflozin and cardiovas cular and renal events in type 2 diabetes. $\mathrm{N}$ Engl J Med 2017; 377: 644-57.

75. Katsiki N, Dimitriadis G, Hahalis G, et al. Sodium-glu cose co-transporter-2 inhibitors (SGLT2i) use and risk of amputation: an expert panel overview of the evidence. Metabolism 2019; 96: 92-100.

76. Wiviott SD, Raz I, Bonaca MP, et al.; DECLARE-TIMI 58 Investigators. Dapagliflozin and cardiovascular outcomes in type 2 diabetes. N Engl J Med 2019; 380: 347-57.

77. Zinman B, Wanner C, Lachin JM, et al.; EMPA-REG OUTCOME Investigators. Empagliflozin, cardiovascular outcomes, and mortality in type 2 diabetes. N Engl J Med 2015; 373: 2117-28.

78. Fitchett D, Inzucchi SE, Cannon CP, et al. empagliflozin reduced mortality and hospitalization for heart failure across the spectrum of cardiovascular risk in the EMPAREG OUTCOME Trial. Circulation 2019; 139: 1384-95.

79. Cosentino F, Ceriello A, Baeres FMM, et al. Addressing cardiovascular risk in type 2 diabetes mellitus: a report from the European Society of Cardiology Cardiovascular Roundtable. Eur Heart J 2019; 40: 2907-19. 